\title{
Modeling and Testing of the Viscoelastic Properties of a Graphite Nanoplatelet/Epoxy Composite
}

\author{
Gregory M. Odegard* \\ Department of Mechanical Engineering - Engineering Mechanics \\ Michigan Technological University \\ 1400 Townsend Drive \\ Houghton, MI 49931
}

\author{
Thomas S. Gates \\ Mechanics and Durability Branch \\ NASA Langley Research Center \\ MS $188 E$ \\ Hampton, VA 23681
}

\begin{abstract}
In order to facilitate the interpretation of experimental data, a micromechanical modeling procedure is developed to predict the viscoelastic properties of a graphite nanoplatelet/epoxy composite as a function of volume fraction and nanoplatelet diameter. The predicted storage and loss moduli for the composite are compared to measured values from the same material using three test methods; Dynamical Mechanical Analysis, nanoindentation, and quasi-static tensile tests. In most cases, the model and experiments indicate that for increasing volume fractions of nanoplatelets, both the storage and loss moduli increase. Also, the results indicate that for nanoplatelet sizes above $15 \mu \mathrm{m}$, nanoindentation is capable of measuring properties of individual constituents of a composite system. Comparison of the predicted values to the measured data helps illustrate the relative similarities and differences between the bulk and local measurement techniques.
\end{abstract}

Keywords: Dynamical Mechanical Analysis, Micromechanics, Modeling, Nanoindentation, Nanotechnology

\section{NOMENCLATURE}

$a_{i} \quad$ Principle half-axes of the inclusion

A Projected contact area

$\mathbf{A}_{p}^{*} \quad$ Complex dilute strain-concentration tensor

$c_{p} \quad$ Volume fraction of nanoplatelets

$c_{m} \quad$ Volume fraction of matrix

C $^{*} \quad$ Complex stiffness tensor of composite

* Corresponding author, email: gmodegar@mtu.edu, phone: (906)487-2329, fax: (906)487-2822 


\begin{tabular}{ll}
$\mathbf{C}_{p}^{*}$ & Complex stiffness tensor of nanoplatelets \\
$\mathbf{C}_{m}^{*}$ & Complex stiffness tensor of matrix \\
$\mathbf{C}^{\prime}$ & Storage tensor of the composite \\
$\mathbf{C}^{\prime \prime}$ & Loss tensor of the composite \\
$D$ & Damping of contact \\
$E^{\prime}$ & Storage modulus \\
$E^{\prime \prime}$ & Loss modulus \\
$G^{\prime}$ & Storage shear modulus \\
$G^{\prime \prime}$ & Loss shear modulus \\
$\mathbf{I}$ & Identity tensor \\
$J_{i}, J_{i j}$ & Eshelby tensor parameters \\
$P$ & Applied nanoindenter force \\
$S$ & Contact stiffness \\
$\mathbf{S}^{*}$ & Complex Eshelby tensor \\
$\mathbf{T}$ & Arbitrary four-order tensor \\
$\tan \delta$ & Loss tangent \\
$x_{i}$ & Nanoplatelet coordinate system \\
$\beta$ & Tip shape constant \\
$\delta_{i j}$ & Kronecker delta \\
$\kappa, \mu$ & Orientation averaging parameters \\
$v^{*}$ & Complex Poisson’s ratio \\
$\omega$ & Harmonic frequency of nanoindenter tip \\
& \\
\hline &
\end{tabular}

\section{INTRODUCTION}

In recent years, nanostructured materials have spurred considerable interest in the materials research community partly because of their potential for large gains in mechanical properties relative to current materials used for aerospace applications. For example, graphite nanoplatelet/polymer composites have been a focus of recent development (Chen, Wu, Weng, 2001; Fukushima and Drzal, 2002; Pan, Yu, Ou, 2000; Thongruang, Balik and Spontak, 2002; Yalcin, Valladares and Cakmak, 2003; Zheng, Wu, Wang, 2004) for use as low-cost, lightweight materials with mechanical properties potentially superior to those of pure polymers without the penalty of increased weight. In order to facilitate the development of these new materials for aerospace applications, modeling and characterization procedures must be developed that address the unique characteristics of nanostructured materials and provide a multi-scale approach that spans the nano- to macro-length scales.

Recently, experiments were conducted to determine the viscoelastic properties of a new nanostructured material: graphite nanoplatelet/epoxy composites (Chasiotis, Chen and Odegard, 2004). The volume fractions and sizes of the nanoplatelets in the test specimens made from this material were varied in a systematic manner to facilitate the exploration of intrinsic material structure on the engineering properties of the composite. To determine these properties, experimental tests were performed that included dynamic nanoindentation, Dynamical 
Mechanical Analysis (DMA), and uniaxial tensile testing. Since these tests were conducted over a wide range of length scales, if follows that the measured elastic and viscoelastic properties for each test must be interpreted based on the relative length scale of the test method and scale of the heterogeneity of the material. However, the correct interpretation and comparison of the experimental data can be difficult without the adequate models to aid in evaluation of properties and behavior.

The objective of the current paper is to develop a viscoelastic modeling procedure to facilitate the interpretation of experimental data and add insight into the measured time-dependent behavior of nano-particle reinforced composites. A brief summary of the material and test methods is provided followed by a detailed explanation of the viscoelastic modeling procedure. The predicted viscoelastic properties from the model are compared to the experimentally obtained properties and the results of this comparison are used to help demonstrate the strengths and limitations of the test methods.

\section{MATERIALS}

In this study, the viscoelastic properties of five materials were examined - four composite materials made from graphite nanoplatelet/epoxy, and one homogeneous material made from unreinforced neat epoxy. All five materials used the same commercial epoxy system. The four composite materials had exfoliated graphite nanoplatelets of two different average diameters, 1 and $15 \mu \mathrm{m}$, for two different nanoplatelet volume fractions, $0.5 \%$ and $3.0 \%$. All the test specimens were fabricated at Michigan State University, and further details on the material, fabrication methods, and initial characterization can be found elsewhere (Fukushima and Drzal, 2002). The variations in volume fraction and platelet size created distinct differences in the relative structure of the composite materials. As an illustrative example of the material structure, Figures 1 and 2 show an Atomic Force Microscope (AFM) image of the $0.5 \%$ nanoplatelet volume fraction composites with $1 \mu \mathrm{m}$ and $15 \mu \mathrm{m}$ diameter nanoplatelets, respectively. Figures 3 and 4 show similar images of the 3.0\% nanoplatelet volume fraction composites with $1 \mu \mathrm{m}$ and $15 \mu \mathrm{m}$ diameter nanoplatelets, respectively. From Figures 1-4, it is clear that the four composite materials have distinct structure and that all materials have the nanoplatelets well dispersed and randomly oriented in the epoxy matrix.

\section{TEST METHODS}

Three test methods were used to determine the mechanical properties of the four composite materials and the neat epoxy material, Dynamical Mechanical Analysis (DMA), quasi-static tensile testing, and nanoindentation. In this section, the basic procedures for each test method is described. Further details of the test methods and apparatus may be found in (Chasiotis, Chen and Odegard, 2004).

\section{Dynamical Mechanical Analysis}

The DMA tests were conducted to determine the dynamic moduli of each material system. These tests were based on well-established procedures (Kampf, 1986; Menard, 1999). The specimens had nominal dimensions of $50 \times 12 \times 5 \mathrm{~mm}$ and were tested using the standard three- 
point bend configuration at room temperature. The tests were conducted with a harmonic amplitude and frequency of $20 \mu \mathrm{m}$ and $50 \mathrm{~Hz}$, respectively. Three tests were performed for each of the five material systems and both storage, $E^{\prime}$, and loss modulus, $E^{\prime \prime}$, were calculated from the measured data. It is important to note that these were bulk-level tests. That is, the measured properties from the DMA represent the overall viscoelastic properties of the entire sample and cannot distinguish the relative influence of each material constituent.

\section{Quasi-Static Tensile Testing}

Quasi-static tensile tests were also performed on bulk samples with the same dimensions as those of the DMA specimens. These tests were used to determine the Young's modulus and Poisson's ratio of each of the specimens. A constant displacement rate of $0.3 \mathrm{~mm} / \mathrm{min}$ was applied to the specimens with a standard tensile-testing machine. A laser extensometer was used to measure the axial strain in the specimen, while strain gages were used to measure the transverse strain. The axial strain was used for the calculation of Young's modulus, and both axial and transverse strains were used for the calculation of Poisson's ratio. The Young's modulus and Poisson's ratio were determined from the initial linear segments of the stress-strain data. Stress was calculated based on the measured load and the specimen's average cross-section prior to test. Three replicates were used for each material.

\section{Nanoindentation}

Dynamic nanoindentation tests were performed at room temperature on samples of the five materials using a previously established procedure (Odegard, Gates and Herring, 2005). The Continuous Stiffness Measurement method, developed by the instrument manufacturer, was used for all tests. This method allowed for a continuous measure of the dynamic stiffness of the material throughout the loading process by using a continuous low magnitude oscillating force superimposed onto the high magnitude quasi-static force applied at the nanoindenter tip. The displacement response was measured at the same frequency as the applied oscillating force, and any resulting phase lag between applied force and measured response was related to the viscous damping of the material using standard concepts of linear viscoelasticty. The harmonic amplitude and frequency of the nanoindenter tip oscillations was $10 \mathrm{~nm}$ and $50 \mathrm{~Hz}$, respectively. The maximum displacement of the tip into the material was limited to $2 \mu \mathrm{m}$. The storage modulus, $E^{\prime}$, and loss modulus, $E^{\prime \prime}$, were determined using the nanoindentation data and the expressions

$$
E^{\prime}=\frac{S}{2 \beta} \sqrt{\frac{P}{A}} \quad E^{\prime \prime}=\frac{\omega D}{2 \beta} \sqrt{\frac{P}{A}}
$$

where $S$ and $D$ are the contact stiffness and damping of contact, respectively, $\omega$ is the harmonic frequency of the nanoindenter tip, $A$ is the projected contact area, $P$ is the applied force, and $\beta$ is the tip-related constant. The values of $S$ and $D$ are determined empirically by calibration of the test apparatus, the projected contact area, $A$, was determined using a method described elsewhere (Odegard, Gates and Herring, 2005), and $\beta$ is equal to 1.034 for the Berkovich tip, which was used in this study. For illustrative purposes, Figure 5 shows an AFM image of the residual 
indentation in the epoxy material after a completed test. Comparison of the size of the indentation in Figure 5 with the sizes of reinforcement in the composite samples in Figures 1-4 reveals that the span of the nanoindentation and the nanoplatelet sizes are similar with both on the $\mu$ m-length scale.

\section{MICROMECHANICS MODEL}

The viscoelastic properties of the four composite materials were predicted by using a micromechanics model that incorporated the individual viscoelastic properties of the graphite and epoxy constituents. The micromechanics-based Mori-Tanaka method (Benveniste, 1987; Mori and Tanaka, 1973) was used in conjunction with the elastic-viscoelastic correspondence principle (Brinson and Lin, 1998). To implement this model for the graphite nanoplatelet/epoxy composite, it was assumed that the nanoplatelets were randomly-oriented, oblate-spheroids that were perfectly bonded to the surrounding matrix (Figure 6). Given these assumptions, the composite complex modulus is

$$
\mathbf{C}^{*}=\left(c_{m} \mathbf{C}_{m}^{*}+c_{p}\left\langle\mathbf{C}_{p}^{*} \mathbf{A}_{p}^{*}\right\rangle\right)\left(c_{m} \mathbf{I}+c_{p}\left\langle\mathbf{A}_{p}^{*}\right\rangle\right)^{-1}
$$

where $c_{p}$ and $c_{m}$ are the nanoplatelet and matrix volume fractions, respectively, $\mathbf{C}_{p}^{*}$ and $\mathbf{C}_{m}^{*}$ are the complex stiffness tensors of the nanoplatelets and matrix, respectively, $\mathbf{I}$ is the identity tensor, the angle-brackets indicate a nanoplatelet-orientation average, and $\mathbf{A}_{p}^{*}$ is the complex dilute strain-concentration tensor of the nanoplatelets. The complex quantities in Equation (2) and throughout this paper can be expressed in terms of real (storage, time-independent) and imaginary (loss, time-dependent) components. For example, the complex stiffness tensor of the composite is

$$
\mathbf{C}^{*}=\mathbf{C}^{\prime}+i \mathbf{C}^{\prime \prime}
$$

where $\mathbf{C}^{\prime}$ and $\mathbf{C}^{\prime \prime}$ are the composite storage and loss stiffness tensors, respectively. The complex dilute strain-concentration tensor in Equation (2) is

$$
\mathbf{A}_{p}^{*}=\left[\mathbf{I}+\mathbf{S}^{*}\left(\mathbf{C}_{m}^{*}\right)^{-1}\left(\mathbf{C}_{p}^{*}-\mathbf{C}_{m}^{*}\right)\right]^{-1}
$$

where $\mathbf{S}^{*}$ is the complex Eshelby tensor. The complex Eshelby tensor is determined by applying the elastic-viscoelastic correspondence principle to the Eshelby tensor for elastic composites (Eshelby, 1957; Mura, 1982), which yields the individual terms for the tensor 


$$
\begin{aligned}
& S_{1111}^{*}=\frac{3}{8 \pi\left(1-v^{*}\right)} a_{1}^{2} J_{11}+\frac{1-2 v^{*}}{8 \pi\left(1-v^{*}\right)} J_{1} \\
& S_{1122}^{*}=\frac{1}{8 \pi\left(1-v^{*}\right)} a_{2}^{2} J_{12}-\frac{1-2 v^{*}}{8 \pi\left(1-v^{*}\right)} J_{1} \\
& S_{1133}^{*}=\frac{1}{8 \pi\left(1-v^{*}\right)} a_{3}^{2} J_{13}-\frac{1-2 v^{*}}{8 \pi\left(1-v^{*}\right)} J_{1} \\
& S_{1212}^{*}=\frac{a_{1}^{2}+a_{2}^{2}}{16 \pi\left(1-v^{*}\right)} J_{12}+\frac{1-2 v^{*}}{16 \pi\left(1-v^{*}\right)}\left(J_{1}+J_{2}\right)
\end{aligned}
$$

where $v^{*}$ is the complex Poisson's ratio; $a_{1}=a_{2}>a_{3}$ are the principle half-axes of the oblate spheroid inclusion (Figure 6); $S_{i j k l}^{*}=S_{j i k l}^{*}=S_{i j k}^{*}$; all other non-zero components are obtained by cyclic permutation of $(1,2,3)$; the components which cannot be obtained by the cyclic permutation are zero; and

$$
\begin{aligned}
& J_{1}=J_{2}=\frac{2 \pi a_{1}^{2} a_{3}}{\left(a_{1}^{2}-a_{3}^{2}\right)^{3 / 2}}\left[\cos ^{-1}\left(\frac{a_{3}}{a_{1}}\right)-\frac{a_{3}}{a_{1}}\left(1-\frac{a_{3}^{2}}{a_{1}^{2}}\right)^{1 / 2}\right] \\
& J_{3}=4 \pi-2 J_{1} \\
& J_{11}=J_{22}=J_{12}=\frac{\pi}{a_{1}^{2}}-\frac{J_{1}-J_{3}}{4\left(a_{3}^{2}-a_{1}^{2}\right)} \\
& J_{13}=J_{23}=\frac{J_{1}-J_{3}}{a_{3}^{2}-a_{1}^{2}} \\
& J_{33}=\frac{1}{3}\left(\frac{4 \pi}{a_{3}^{2}}-2 J_{13}\right)
\end{aligned}
$$

For three-dimensional, randomly-oriented, nanoplatelet composite materials, the orientation average of an arbitrary forth-order tensor, $\mathbf{T}$, associated with the isotropic material symmetry is

$$
\langle\mathbf{T}\rangle=\left\langle T_{i j k l}\right\rangle=\left(\kappa-\frac{2}{3} \mu\right)\left(\delta_{i j} \delta_{k l}\right)+\mu\left(\delta_{i k} \delta_{j l}+\delta_{i l} \delta_{j k}\right)
$$

where $i, j, k, l=1,2,3$; the indicial summation convention is used; $\delta_{i j}$ is the Kronecker delta; and

$$
\begin{gathered}
\kappa=\frac{1}{9} T_{i i j j} \\
\mu=\frac{1}{10}\left(T_{i j i j}-\frac{1}{3} T_{i i j j}\right)
\end{gathered}
$$


Therefore, from Equations (7) and (8), $\langle\mathbf{T}\rangle$ is isotropic.

The properties of the constituent materials used in this model are shown in Table 1. It was assumed that the epoxy matrix was isotropic, with $C_{11}^{*}=C_{22}^{*}=C_{33}^{*}, C_{23}^{*}=C_{13}^{*}=C_{12}^{*}$, and $C_{44}^{*}=C_{55}^{*}=C_{66}^{*}$. It was also assumed that the graphite nanoplatelets were linear-elastic, with the classic transversely-isotropic graphite mechanical properties being used (Kelly, 1981), where the $x_{3}$ axis is perpendicular to the graphene plane. The storage and loss moduli, $E^{\prime}$ and $E^{\prime \prime}$, respectively, of the epoxy material were those determined via DMA testing described previously. It was assumed that the storage shear modulus of the epoxy, $G^{\prime}$, was equal to the static shear modulus, which was computed using the Young's modulus and Poisson's ratio determined via the quasi-static tensile testing. The loss shear modulus, $G^{\prime \prime}$, was determined using (Ferry, 1980)

$$
\tan \delta=\frac{E^{\prime \prime}}{E^{\prime}}=\frac{G^{\prime \prime}}{G^{\prime}}
$$

With these assumptions, it was possible to uniquely determine all of the components of the complex stiffness tensors of the nanoplatelets and polymer using the elastic-viscoelastic correspondence principle.

For the complex Eshelby tensor, the complex material properties of the epoxy and the assumed shape of the nanoplatelets were used in Equations (5) and (6). It was assumed that the principle half-axes $a_{1}$ and $a_{2}$ were equal to half of the total length (radius) of the nanoplatelets. Therefore, for the $1 \mu \mathrm{m}$-sized and $15 \mu \mathrm{m}$-sized nanoplatelets, $a_{1}$ and $a_{2}$ were $0.5 \mu \mathrm{m}$ and $7.5 \mu \mathrm{m}$, respectively. From AFM images of the material, such as that shown in Figures 1-4, it was determined that the graphite nanoplatelets had a thickness of about $0.5 \mu \mathrm{m}$ for the $1 \mu \mathrm{m}$ nanoplatelets and $1 \mu \mathrm{m}$ for the $15 \mu \mathrm{m}$ nanoplatelets. Therefore, $a_{3}$ was $0.25 \mu \mathrm{m}$ and $0.5 \mu \mathrm{m}$ for the $1 \mu \mathrm{m}$ and $15 \mu \mathrm{m}$-sized nanoplatelets, respectively.

At this point, some of the possible influences of the model assumptions are discussed. First, previous studies have shown that the mechanical properties of composites with nanometer-sized reinforcement can be modeled using a multi-scale equivalent-continuum modeling approach (Odegard, Frankland and Gates, 2003; Odegard, Gates, Nicholson, 2002; Odegard, Gates, Wise, 2003). This type of modeling approach requires coupled atomistic and continuum modeling techniques. However, because the smallest dimension of the nanoplatelet is $0.5 \mu \mathrm{m}$, which has been demonstrated to be large enough to be modeled directly using micromechanics (Odegard, Clancy and Gates, 2005), the fully coupled approach is not employed in the current research. Secondly, it is recognized that the assumption of perfect bonding between the nanoplatelets and epoxy matrix could potentially lead to discrepancies in the magnitudes of moduli determined from modeling and experiment. However, because the relative quality of the actual bonding between the reinforcement and matrix in the fabricated specimens is not known, the simple assumption of perfect bonding was adopted. This assumption should not influence the trends in the predicted moduli data with respect to reinforcement size and volume fraction. 


\section{RESULTS AND DISCUSSION}

The bulk and localized storage moduli of the neat epoxy and four composite materials measured in the DMA and nanoindentation experiments and the associated values predicted by the model are shown in Figure 7. For completeness, the Young's moduli of the neat epoxy and three of the composite materials as measured via the tensile tests are also shown in Figure 7. The bulk and localized loss moduli of the composite materials measured through DMA and nanoindentation experiments and the corresponding values predicted by the model are shown in Figure 8. The error bars shown for the experimental data in Figures 7 and 8 represent the standard deviation of the mean (standard error) for the repeat tests.

The results presented in Figure 7 indicate that there is reasonable agreement between storage modulus for all of the experimental tests. For all three experimental sets of data, the storage modulus (or Young's modulus for the tensile tests) increases with an increasing nanoplatelet volume fraction, as expected. This trend is confirmed by results from studies on graphite platelet/Polyethylene composites (Thongruang, Balik and Spontak, 2002) and graphite nanoplatelet/epoxy composites (Fukushima and Drzal, 2002). The only exception to this observation is the $0.5 \%$ volume fraction specimen with $15 \mu \mathrm{m}$ nanoplatelets, for which the storage modulus from nanoindentation is about equal to that of the epoxy material. The model confirms this overall trend, although the magnitudes of the predicted values of storage modulus are lower than the measured values. Because of the lower magnitudes of the predicted storage moduli, it appears that for the $0.5 \%$ volume fraction specimen with $1 \mu \mathrm{m}$ nanoplatelets, the model shows closer agreement with the tensile test and DMA data than the nanoindentation data. For the other three composites, it appears that the predicted storage moduli have the closest agreement with the nanoindentation tests. This does not necessarily indicate that, in general, the model predicts storage moduli that are in closer agreement with the nanoindentation data, but that the magnitudes of the predicted data tend to be lower than those of the experimental data, while the trends are similar.

For the DMA and tensile test data, representing bulk measurements, there is no clear effect of increasing the nanoparticle diameter on the measured storage modulus, given the standard error of the experiments. Similarly, there is no clear trend in the predicted data for increases in nanoparticle diameter. The nanoindentation data, representing localized measurements, shows a very clear decrease in measured storage modulus with increasing nanoplatelet diameter. For the $1 \mu \mathrm{m}$ diameter nanoplatelets, the storage modulus values from the nanoindentation are very close to those measured with the DMA. For the $15 \mu \mathrm{m}$-diameter nanoplatelets, the measured storage moduli from nanoindentation are close to those of the epoxy material.

All of these results in reference to Figure 7 lead to the assumption that because the DMA, tensile test, and modeling data represent bulk-level behavior of the composite materials, and the nanoindentation data is the result of local-level behavior (on the $\mu \mathrm{m}$-length scale), the nanoindenter must be sampling mostly the bulk polymer material in the $15 \mu \mathrm{m}$-diameter specimens, and sampling a mixture of polymer and nanoplatelet reinforcement in the $1 \mu \mathrm{m}$ diameter nanoplatelets specimens. To substantiate this assumption consider that for a given nanoplatelet volume fraction, a $15 \mu \mathrm{m}$-sized indentation tip (Figure 5) has a higher probability of indenting a pure polymer material with $15 \mu \mathrm{m}$-diameter nanoplatelets than with $1 \mu \mathrm{m}$-diameter 
nanoplatelets. Therefore, for the nanoindenter tip size, tip geometry, and nanoplatelet volume fractions considered in this study, composites materials with nanoplatelet sizes below $1 \mu \mathrm{m}$ represent a statistically homogeneous medium for nanoindentation testing. Conversely, for composite materials with nanoplatelet sizes above $15 \mu \mathrm{m}$, nanoindentation is capable of examining the individual phases in the material thereby providing data on localized properties.

Examining the data in Figure 8, it is clear that the loss moduli measured with nanoindentation are at least 50\% higher than those measured with the DMA for the four composite materials and the epoxy. Furthermore, the predicted values are in much better agreement with the DMA data than for the nanoindentation data. The latter trend is expected because the model used as input the epoxy viscoelastic properties obtained from DMA tests.

While the experimental data show an increase in loss modulus with increased nanoplatelet volume fraction, the model does not predict this trend with the $1 \mu \mathrm{m}$ nanoplatelets. Therefore, this disagreement between experiment and modeling indicate that the model does not correctly establish the necessary relationship between loss modulus and nanoplatelet volume fraction for these materials. However, both experiments and model indicate an increase in loss modulus with increased nanoplatelet diameter for a given nanoplatelet volume fraction. When comparing the test data to the predicted values, it is also useful to note that the room temperature loss modulus for these materials is substantially lower than the corresponding storage modulus and therefore represents data at the lower bound of the resolution for the instrumentation.

\section{SUMMARY}

In this study, a micromechanical model based on the Mori-Tanaka method and the viscoelastic correspondence principle was developed to predict the viscoelastic properties of graphite nanoplatelet/epoxy composites as a function of platelet volume fraction and diameter. Bulk static and viscoelastic properties were measured for all materials using tensile tests and dynamic mechanical analysis (DMA) tests methods respectively. Local viscoelastic properties were measured using nanoindentation test methods. The predicted storage and loss modulus was compared to the experimentally measured viscoelastic and static properties and thus facilitated the interpretation of the differences between local and bulk measurement techniques.

The predicted results and all three experimental techniques indicate that for increasing volume fraction of nanoplatelets, the storage modulus increases, as expected. Based on the bulk properties from DMA, tensile tests, and comparing to model predictions, there is no clear relationship between nanoparticle diameter and storage modulus. The results also indicate that for composites materials with nanoplatelet diameters below $1 \mu \mathrm{m}$, localized nanoindentation tests measure storage moduli that are equal to those of the bulk composite, while for composites with nanoplatelet sizes above $15 \mu \mathrm{m}$, and given the relatively low volume fraction of reinforcement, nanoindentation measurements closely match the properties of the epoxy material.

Therefore, for nanostructured composite materials with local reinforcement size on the order of $15 \mu \mathrm{m}$ or greater, the nanoindentation test method used in this study may be suitable for providing localized material properties associated with individual phases or constituents. This 
type of practical limit on resolution and the relationship to material structure (i.e. size of reinforcement and volume fraction), illustrates the need for careful selection of appropriate modeling and measurement methods when characterizing the local behavior of nanostructured materials.

\section{ACKNOWLEDGEMENTS}

The Authors would like to thank Professor Ioannis Chasiotis of the Department of Aerospace Engineering at the University of Illinois at Urbana-Champaign for providing the AFM images of the materials.

\section{REFERENCES}

Benveniste, Y. 1987. "A New Approach to the Application of Mori-Tanaka's Theory in Composite Materials," Mechanics of Materials, 6:147-157

Brinson, L.C. and W.S. Lin. 1998. "Comparison of Micromechanics Methods for Effective Properties of Multiphase Viscoelastic Composites," Composite Structures, 41:353-367

Chasiotis, I., Q. Chen and G.M. Odegard. "Multiscale Experiments on Graphite Nanoplatelet/Epoxy Composites," SEM X International Congress and Exposition on Experimental and Applied Mechanics. Costa Mesa, CA, 2004.

Chen, G.H., D.J. Wu, W.G. Weng and W.L. Yan. 2001. "Preparation of Polymer/Graphite Conducting Nanocomposite by Intercalation Polymerization," Journal of Applied Polymer Science, 82:2506-2513

Eshelby, J.D. 1957. "The Determination of the Elastic Field of an Ellipsoidal Inclusion, and Related Problems," Proceedings of the Royal Society of London, Series A, 241:376-396

Ferry, J.D. 1980. Viscoelastic Properties of Polymers. John Wiley \& Sons, Inc. New York

Fukushima, H. and L.T. Drzal. "Graphite Nanoplatelets as Reinforcements for Polymers: Structural and Electrical Properties," 17th Annual American Society for Composites Technical Conference. West Lafayette, IN, 2002.

Kampf, G. 1986. Characterization of Plastics by Physical Methods: Experimental Techniques and Practical Application. Hanser Publishers. New York

Kelly, B.T. 1981. Physics of Graphite. Applied Science Publishers. Essex, England

Menard, K.P. 1999. Dynamic Mechanical Analysis: A Practical Introduction. CRC Press. Boca Raton, FL

Mori, T. and K. Tanaka. 1973. "Average Stress in Matrix and Average Elastic Energy of Materials with Misfitting Inclusions," Acta Metallurgica, 21:571-574

Mura, T. 1982. Micromechanics of Defects in Solids. Martinus Nijhoff. The Hague

Odegard, G.M., T.C. Clancy and T.S. Gates. 2005. "Modeling the Mechanical Properties of nanoparticle/polymer composites," Polymer, 46:553-562

Odegard, G.M., S.J.V. Frankland and T.S. Gates. "The Effect of Chemical Functionalization on Mechanical Properties of Nanotube/Polymer Composites," 44th 
AIAA/ASME/ASCE/AHS Structures, Structural Dynamics, and Materials Conference. Norfolk, VA, 2003.

Odegard, G.M., T.S. Gates and H.M. Herring. 2005. "Characterization of Viscoelastic Properties of Polymeric Materials Through Nanoindentation," Experimental Mechanics, in press:

Odegard, G.M., T.S. Gates, L.M. Nicholson and K.E. Wise. 2002. "Equivalent-Continuum Modeling of Nano-Structured Materials," Composites Science and Technology, 62:18691880

Odegard, G.M., T.S. Gates, K.E. Wise, C. Park and E. Siochi. 2003. "Constitutive Modeling of Nanotube-Reinforced Polymer Composites," Composites Science and Technology, 63:1671-1687

Pan, Y.X., Z.Z. Yu, Y.C. Ou and G.H. Hu. 2000. "A New Process of Fabricating Electrically Conducting Nylon 6/Graphite Nanocomposites via Intercalation Polymerization," Journal of Polymer Science Part B: Polymer Physics, 38:1626-1633

Thongruang, W., C.M. Balik and R.J. Spontak. 2002. "Volume-Exclusion Effects in Polyethylene Blends Filled with Carbon Black, Graphite, or Carbon Fiber," Journal of Polymer Science Part B: Polymer Physics, 40:1013-1023

Yalcin, B., D. Valladares and M. Cakmak. 2003. "Amplification Effect of Platelet Type Nanoparticles on the Orientation Behavior of Injection Molded Nylon 6 Composites," Polymer, 44:6913-6925

Zheng, G.H., J.S. Wu, W.P. Wang and C.Y. Pan. 2004. "Characterization of Expanded Graphite/Polymer Composites Prepared by In Situ Polymerization," Carbon, 42:28392847 
Journal of Intelligent Materials Systems and Structures, Vol. 17, no. 3, pp. 239-246 (2006)

Table 1. Viscoelastic properties of constituent materials in GPa

\begin{tabular}{ccc}
\hline Property & Graphite & Epoxy \\
\hline$C_{11}^{*}$ & 106.00 & $4.30+0.16 i$ \\
$C_{12}^{*}$ & 18.00 & $2.12+0.08 i$ \\
$C_{13}^{*}$ & 1.50 & $2.12+0.08 i$ \\
$C_{33}^{*}$ & 3.65 & $4.30+0.16 i$ \\
$C_{44}^{*}$ & 0.45 & $1.09+0.04 i$ \\
\hline
\end{tabular}




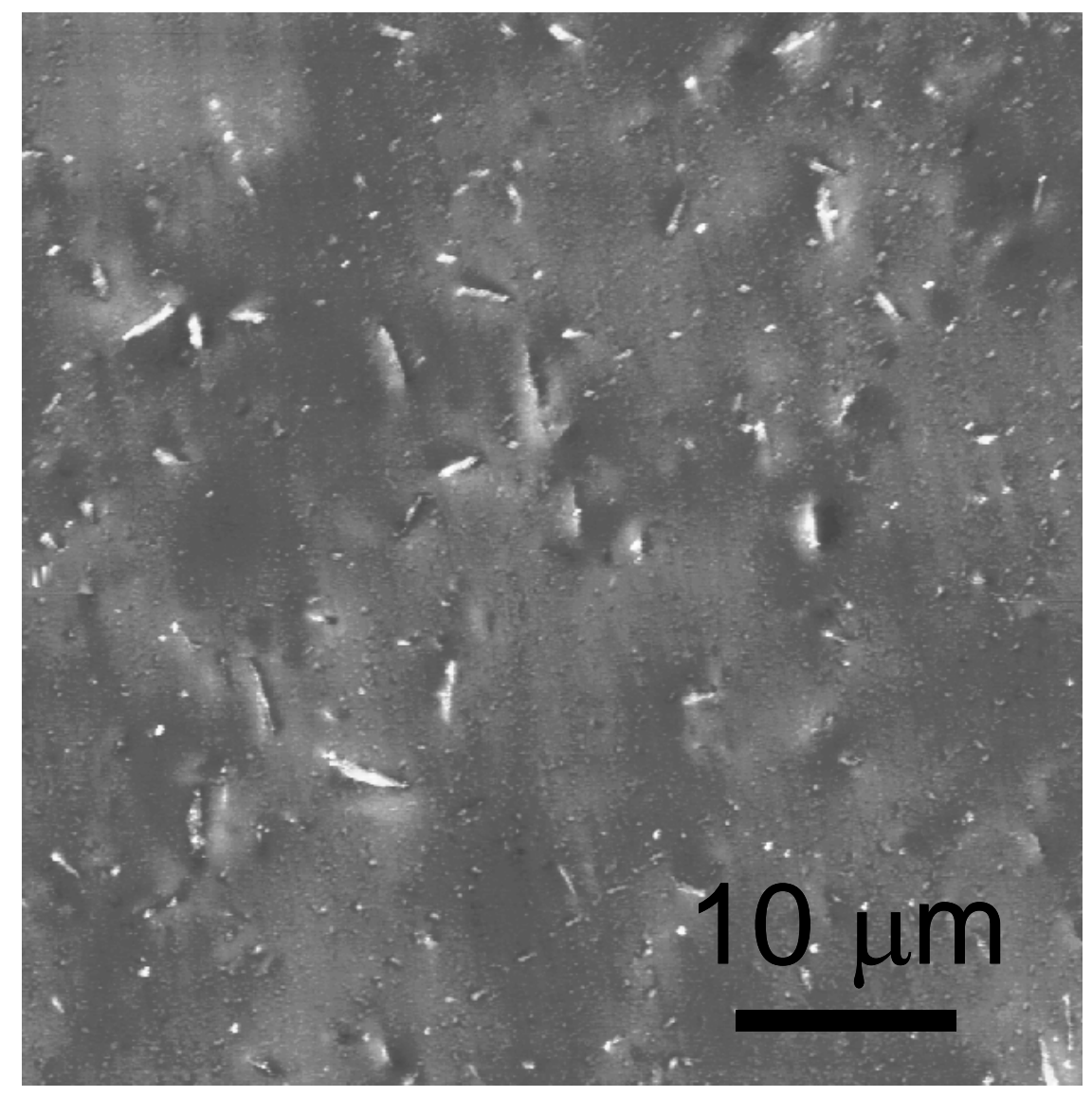

Figure 1. AFM image of graphite nanoplatelet/epoxy composite with a $0.5 \%$ volume fraction of $1 \mu \mathrm{m}$ diameter nanoplatelets 
Journal of Intelligent Materials Systems and Structures, Vol. 17, no. 3, pp. 239-246 (2006)

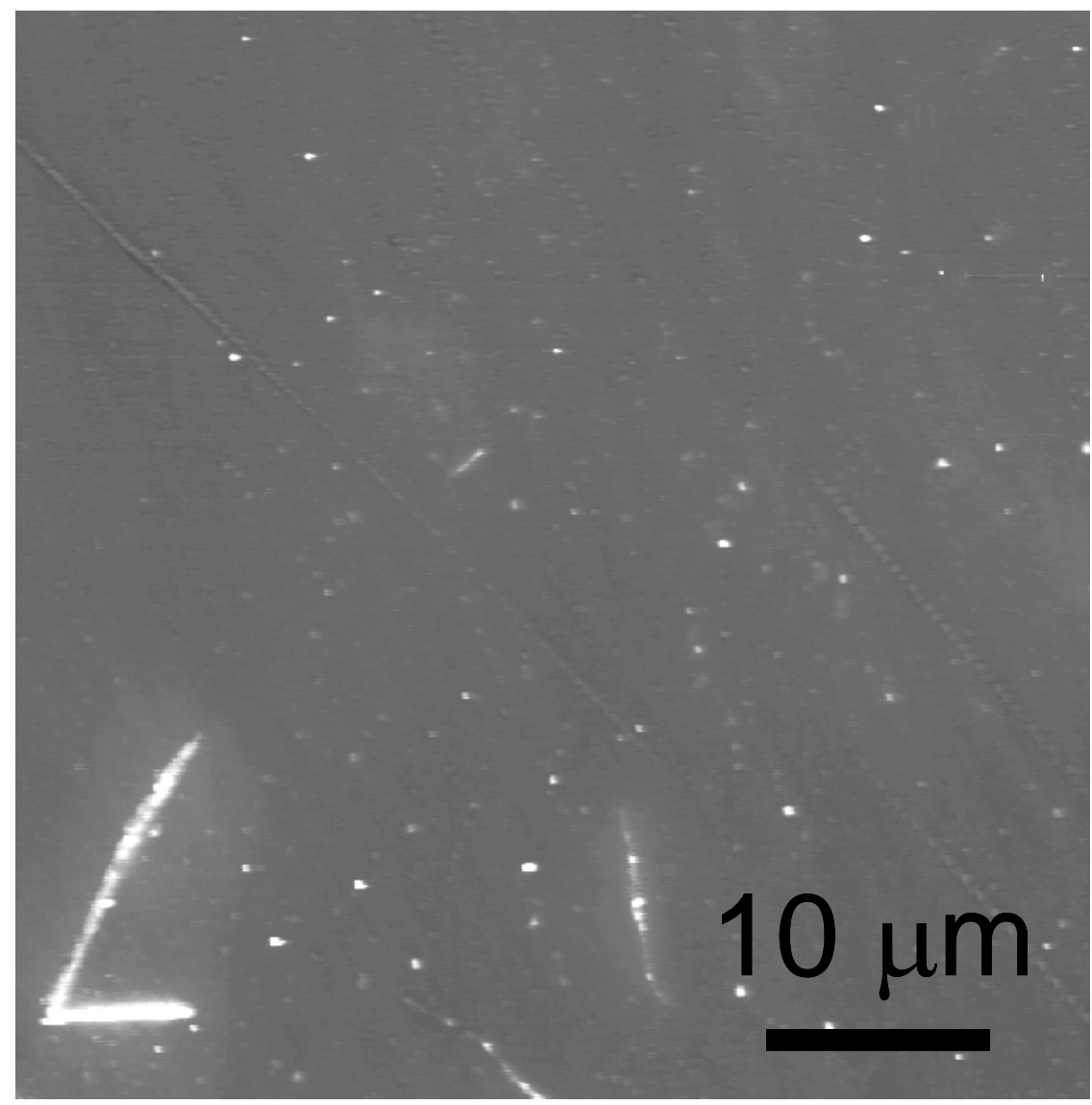

Figure 2. AFM image of graphite nanoplatelet/epoxy composite with a $0.5 \%$ volume fraction of $15 \mu \mathrm{m}$ diameter nanoplatelets 
Journal of Intelligent Materials Systems and Structures, Vol. 17, no. 3, pp. 239-246 (2006)

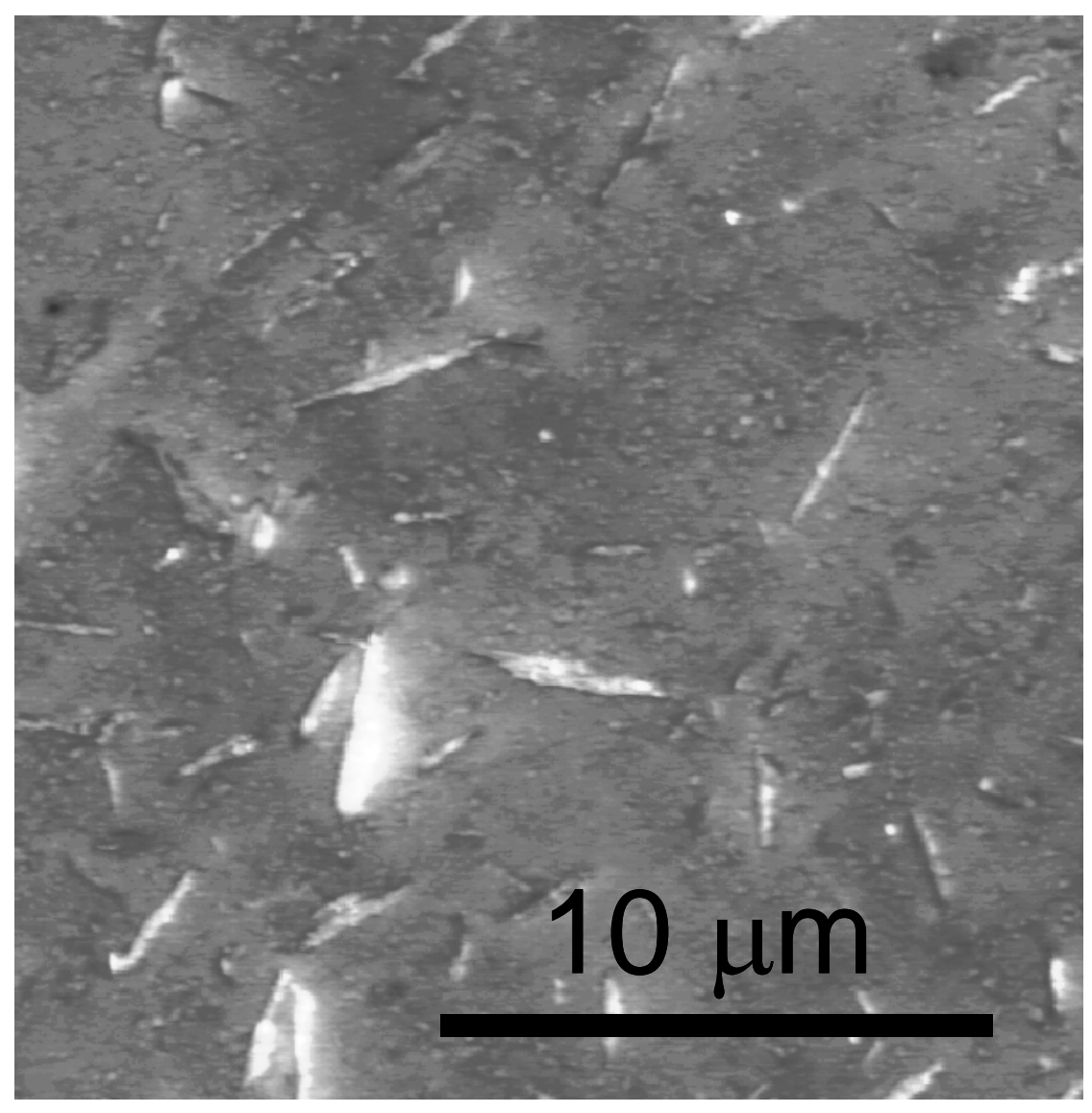

Figure 3. AFM image of graphite nanoplatelet/epoxy composite with a 3.0\% volume fraction of $1 \mu \mathrm{m}$ diameter nanoplatelets 


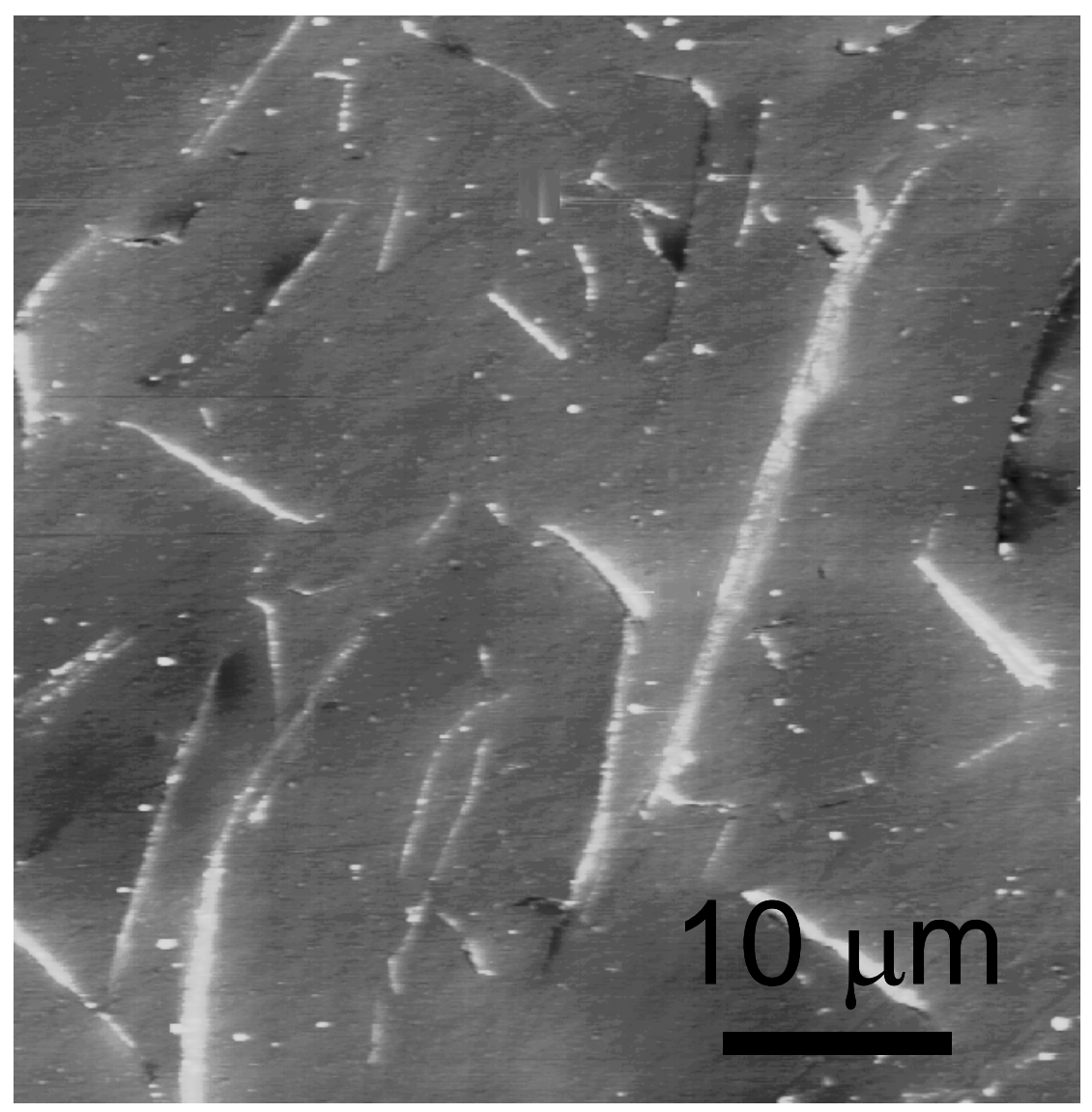

Figure 4. AFM image of graphite nanoplatelet/epoxy composite with a 3.0\% volume fraction of $15 \mu \mathrm{m}$ diameter nanoplatelets 


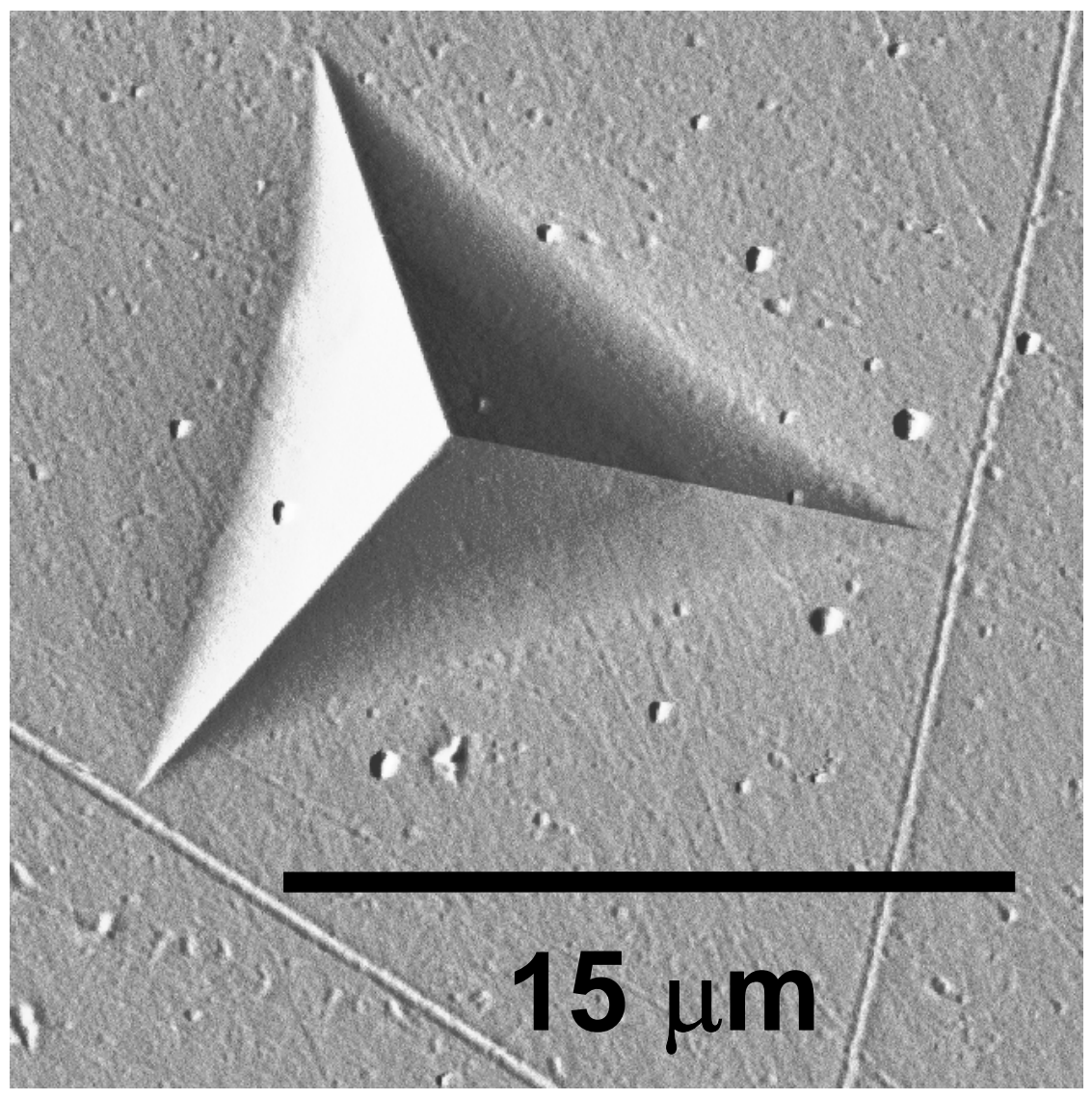

Figure 5. AFM image of nanoindentation site in the epoxy material 


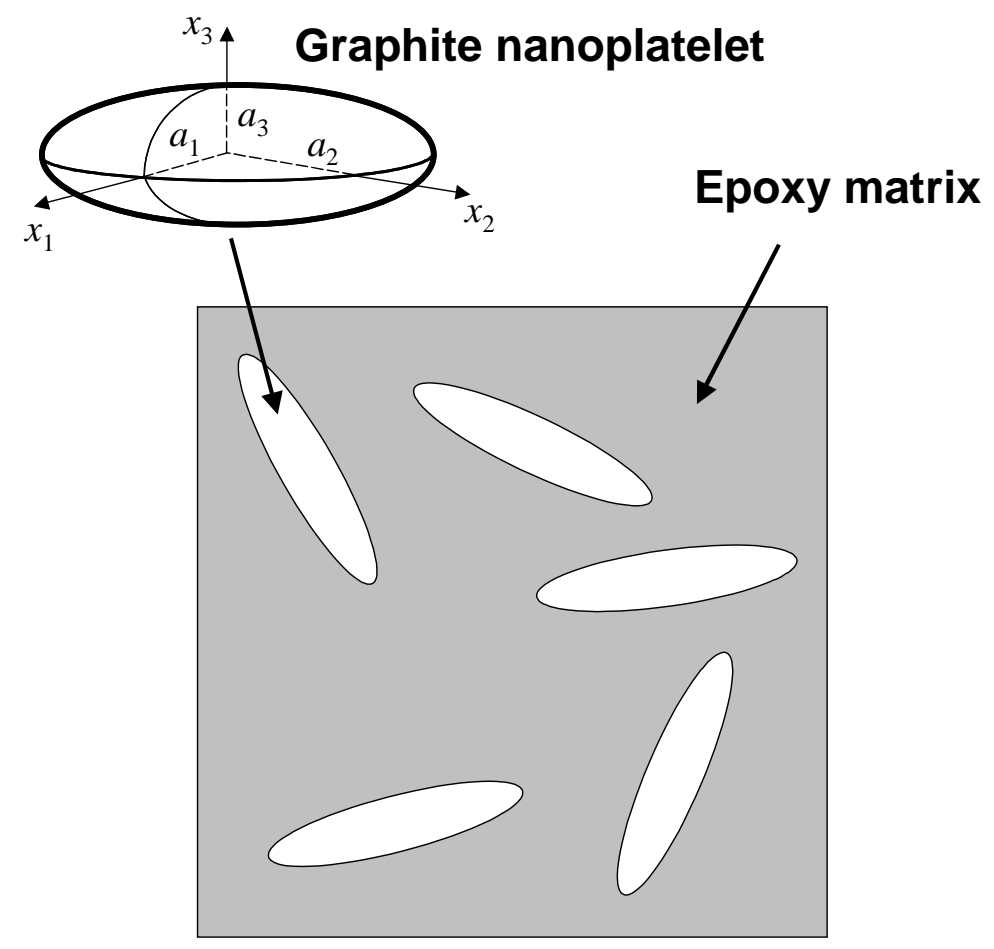

Figure 6. Micromechanical modeling of graphite nanoplatelet/epoxy composite 


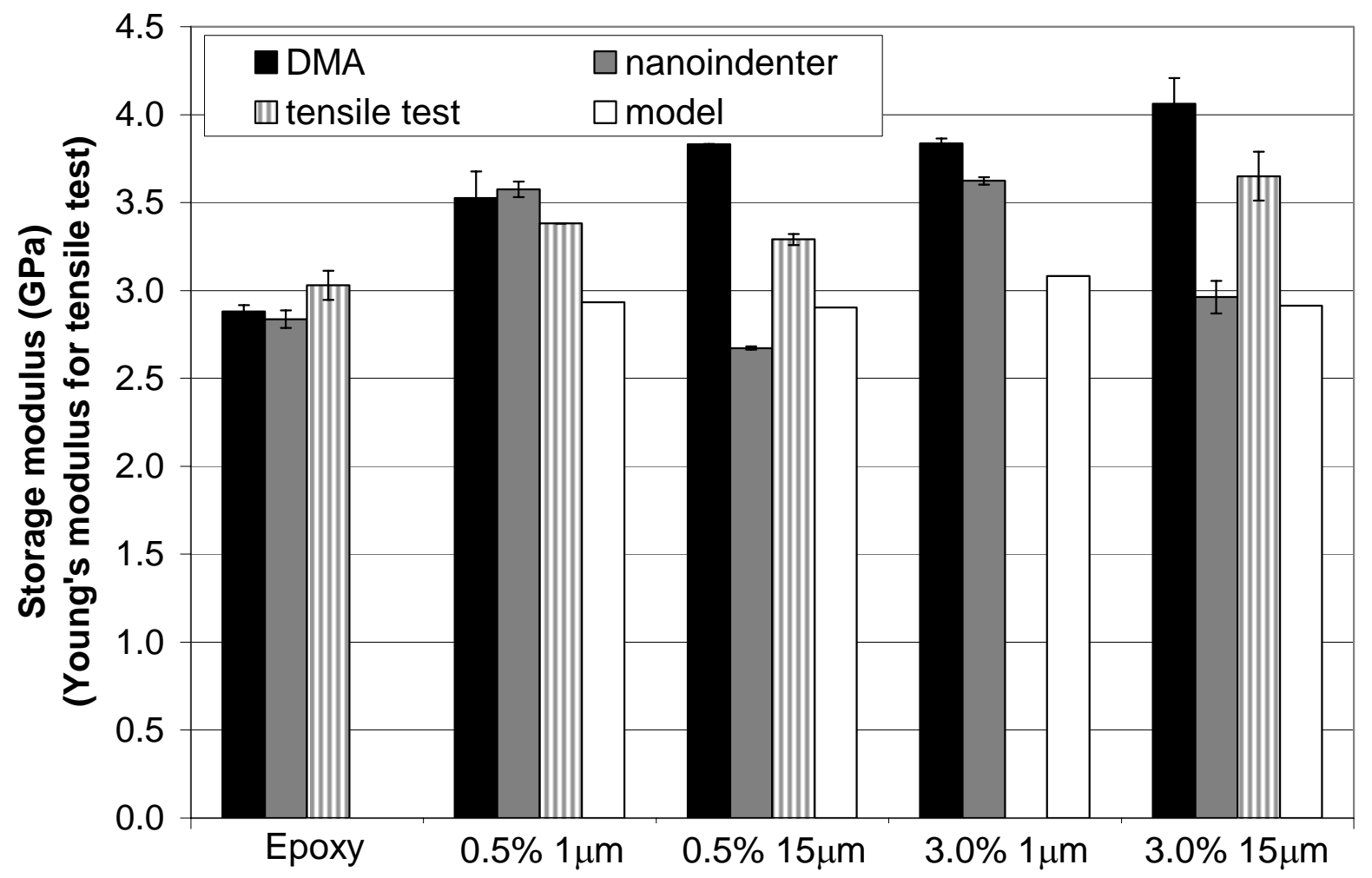

Figure 7. Storage and Young's moduli for graphite nanoplatelet/epoxy composites. (Note that tensile test data for the $3.0 \%, 1 \mu \mathrm{m}$ data is not available) 


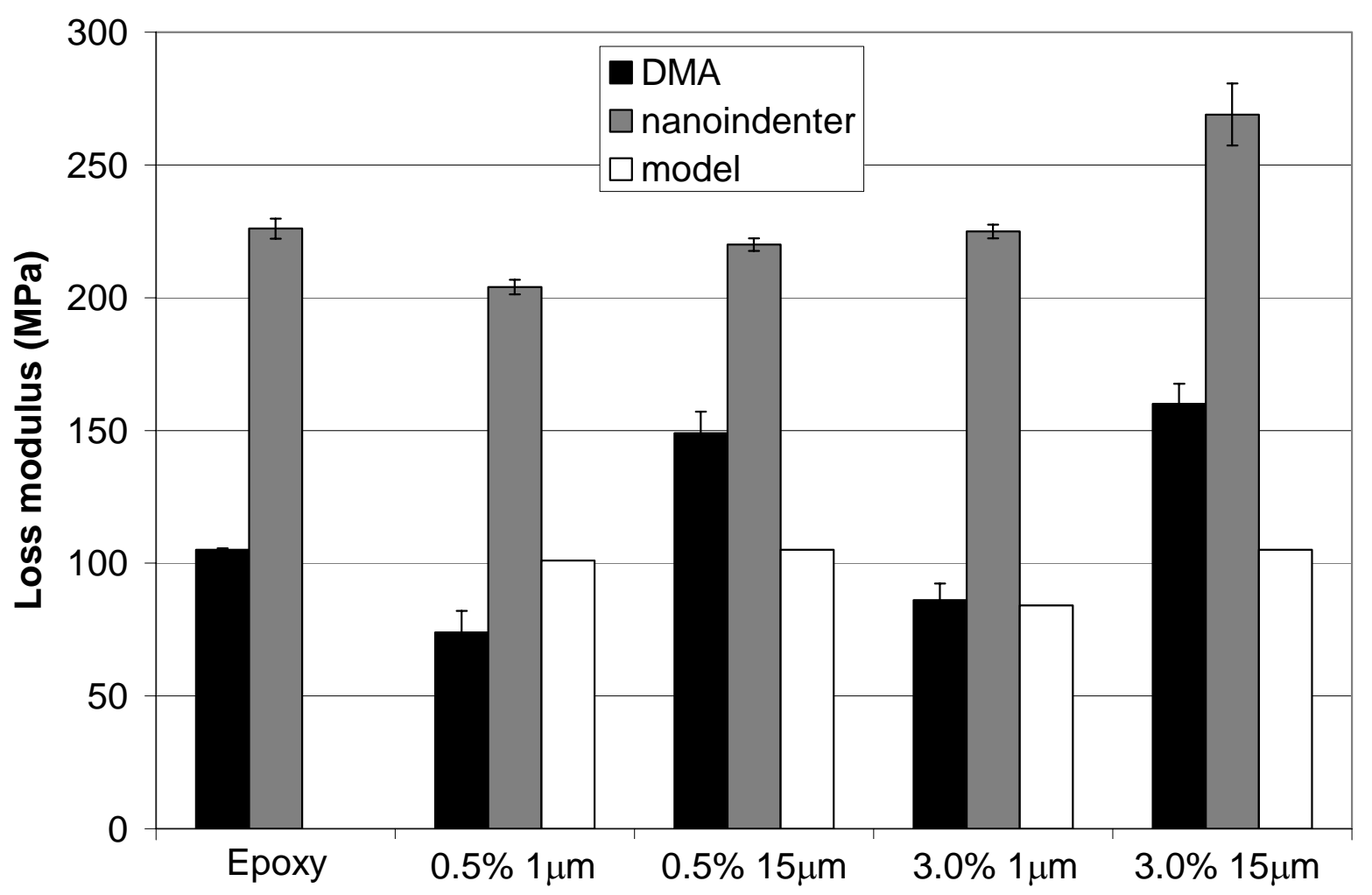

Figure 8. Loss moduli for graphite nanoplatelet/epoxy composites 